\title{
Fractional differential equations and inclusions with semiperiodic and three-point boundary conditions
}

\author{
Bashir Ahmad ${ }^{1 *}$, Ravi P Agarwal ${ }^{2}$ and Ahmed Alsaedi ${ }^{1}$
}

\author{
"Correspondence: \\ bashirahmad_qau@yahoo.com \\ ${ }^{1}$ Nonlinear Analysis and Applied \\ Mathematics (NAAM) Research \\ Group, Department of Mathematics, \\ Faculty of Science, King Abdulaziz \\ University, P.O. Box 80203, Jeddah, \\ 21589, Saudi Arabia \\ Full list of author information is \\ available at the end of the article
}

\begin{abstract}
In this article, we investigate the existence of solutions for boundary value problems of fractional differential equations and inclusions with semiperiodic and three-point boundary conditions. The existence results for equations are obtained by applying Banach's contraction mapping principle, Schaefer-type fixed point theorem, Leray-Schauder degree theory, Krasnoselskii's fixed point theorem, and Leray-Schauder nonlinear alternative, whereas the existence of solutions for convex and nonconvex set-valued maps (inclusion case) is shown via nonlinear alternative of Leray-Schauder type for multivalued maps and Wegrzyk's fixed point theorem for generalized contractions, respectively. We emphasize that a variety of fixed point theorems are used to obtain different existence criteria for the problems at hand. Several examples are discussed for illustration of the obtained results. Moreover, an interesting observation related to symmetric second-order three-point boundary value problems is presented.
\end{abstract}

MSC: 34A08; 34A60; 34B15

Keywords: fractional differential equations; semi-periodic; three-point; boundary conditions; existence; fixed point

\section{Introduction}

In this paper, we study a boundary value problem of nonlinear fractional differential equations with semiperiodic and three-point boundary conditions given by

$$
\begin{aligned}
& { }^{c} D^{q} x(t)+f(t, x(t))=0, \quad t \in[0,1], 1<q \leq 2, \\
& x(0)=x(1), \quad \xi x^{\prime}(0)-\eta x^{\prime}(1)=\zeta x(1 / 2),
\end{aligned}
$$

where $f$ is a given continuous function, and $\xi, \eta, \zeta$ are positive real constants with $\frac{\xi}{\zeta}>$ $\left(\frac{1}{2}+\frac{\eta}{\zeta}\right)$. As a companion problem, we also discuss the inclusion case of problem (1.1).

Multipoint nonlocal boundary value problems of ordinary, integro-differential, and partial differential equations have been extensively studied, and a variety of results can be found in the recent literature, for instance, see [1-9] and the references therein.

The subject of fractional differential equations and inclusions has attracted the attention of many researchers in the last decade. One of the reasons for popularity of the sub-

(c) 2016 Ahmad et al. This article is distributed under the terms of the Creative Commons Attribution 4.0 International License (http://creativecommons.org/licenses/by/4.0/), which permits unrestricted use, distribution, and reproduction in any medium, provided you give appropriate credit to the original author(s) and the source, provide a link to the Creative Commons license, and indicate if changes were made. 
ject is that fractional-order differential and integral operators are nonlocal in nature and help to trace the past history of several materials and processes. For a detailed account of the results on nonlocal fractional-order boundary value problems, we refer the reader to [10-20] and the references therein. Concerning the application of fractional calculus, we can notice that fractional differential equations have played a key role in improving the mathematical modeling of several phenomena occurring in basic technical sciences and engineering. As a matter of fact, this subject is gaining more and more popularity due to its widespread applications. For examples and details, see [21-25].

The paper is organized as follows. Section 2 contains existence and uniqueness results for problem (1.1). These results are new and rely on Banach's contraction mapping principle, a Schaefer-type fixed point theorem, Leray-Schauder degree theory, Krasnoselskii's fixed point theorem, and Leray-Schauder nonlinear alternative. In Section 3, we introduce a multivalued analog of problem (1.1) and prove the existence of solutions for convex setmaps by means of a multivalued version of nonlinear alternative of Leray-Schauder type, whereas the existence result for nonconvex set-valued maps is established by applying Wegrzyk's theorem for generalized contractions. It is important to mention that the existence result obtained by means of Wegrzyk's theorem holds for several choices of the strictly increasing function involved in its hypothesis, in contrast to that obtained by applying a fixed point theorem due to Covitz and Nadler [26]. We emphasize that the exposition of the standard tools of fixed point theory in the framework of the present work is new.

\section{Preliminaries}

Definition 2.1 ([21]) The Riemann-Liouville fractional integral of order $r$ for a continuous function $h$ is defined as

$$
I^{r} h(t)=\frac{1}{\Gamma(r)} \int_{0}^{t} \frac{h(s)}{(t-s)^{1-r}} d s, \quad r>0,
$$

provided that the integral exists.

Definition 2.2 ([21]) For at least $n$ times absolutely continuously differentiable function $h:[0, \infty) \rightarrow \mathbb{R}$, the Caputo derivative of fractional order $r$ is defined as

$$
{ }^{c} D^{r} h(t)=\frac{1}{\Gamma(n-r)} \int_{0}^{t}(t-s)^{n-r-1} h^{(n)}(s) d s, \quad n-1<r<n, n=[r]+1,
$$

where $[r]$ denotes the integer part of a real number $r$.

Lemma 2.3 ([22]) Let $u \in A C^{m}[0,1]$ and $v \in A C[0,1]$. Then, for $\rho \in(m-1, m), m \in \mathbb{N}$, and $t \in[0,1]$,

(a) the general solution of the fractional differential equation ${ }^{c} D^{\rho} u(t)=0$ is given by

$$
u(t)=b_{0}+b_{1} t+b_{2} t^{2}+\cdots+b_{m-1} t^{m-1},
$$

where $b_{i} \in \mathbb{R}, i=0,1,2, \ldots, m-1$;

(b) $I^{\rho c} D^{\rho} u(t)=u(t)-\sum_{k=0}^{m-1} \frac{t^{k}}{k !} u^{k}(0)$;

(c) ${ }^{c} D^{\rho} I^{\rho} v(t)=v(t)$. 
Remark 2.4 Let $A C^{2}[0,1]=\left\{x \in C^{1}[0,1]: x^{\prime} \in A C[0,1]\right\}$. Then $A C^{2}[0,1]=\left\{x \in C^{1}[0,1]\right.$ : $\left.x^{\prime \prime} \in L[0,1]\right\}$. Observe that

$$
{ }^{c} D^{r} h(t)=\frac{1}{\Gamma(2-r)} \int_{0}^{t}(t-s)^{(2-r)-1} h^{\prime \prime}(s) d s=I^{2-r} h^{\prime \prime}(t)=\left(I^{2-r} h(t)\right)^{\prime \prime}, \quad 1<r<2 .
$$

Therefore, ${ }^{c} D^{r}$ maps $A C^{2}[0,1] \subset L(0,1)$ into $L(0,1)$ for $r \in(1,2)$. For more details on Riemann-Liouville fractional integrals, see $[21,22]$.

The following lemma plays a pivotal role to define the solutions for the given problem.

Lemma 2.5 Let $g \in A C[0,1]$ and $x \in A C^{2}[0,1]$. Then the problem

$$
\begin{aligned}
& { }^{c} D^{q} x(t)+g(t)=0, \quad t \in[0,1], 1<q \leq 2, \\
& x(0)=x(1), \quad \xi x^{\prime}(0)-\eta x^{\prime}(1)=\zeta x(1 / 2),
\end{aligned}
$$

is equivalent to the fractional integral equation

$$
\begin{aligned}
x(t)= & -\int_{0}^{t} \frac{(t-s)^{q-1}}{\Gamma(q)} g(s) d s+\left(t-\frac{1}{2}+\frac{\xi-\eta}{\zeta}\right) \int_{0}^{1} \frac{(1-s)^{q-1}}{\Gamma(q)} g(s) d s \\
& +\int_{0}^{\frac{1}{2}} \frac{\left(\frac{1}{2}-s\right)^{q-1}}{\Gamma(q)} g(s) d s+\frac{\eta}{\zeta} \int_{0}^{1} \frac{(1-s)^{q-2}}{\Gamma(q-1)} g(s) d s .
\end{aligned}
$$

Proof By Lemma 2.3, the solution of the fractional differential equation (2.1) can be written as

$$
x(t)=-\int_{0}^{t} \frac{(t-s)^{q-1}}{\Gamma(q)} g(s) d s+b_{1}+b_{2} t
$$

where $b_{1}, b_{2} \in \mathbb{R}$ are unknown arbitrary constants. Using the first condition given by (2.2) in (2.4) gives

$$
b_{2}=\int_{0}^{1} \frac{(1-s)^{q-1}}{\Gamma(q)} g(s) d s
$$

From (2.4), for $t \in[0,1]$, we have

$$
x^{\prime}(t)=-\int_{0}^{t} \frac{(t-s)^{q-2}}{\Gamma(q-1)} g(s) d s+b_{2}=-I^{q-1} g(t)+b_{2}
$$

As argued in [16], for $q-1 \in(0,1), I^{q-1}$ maps $L(0,1)$ into $F_{0}^{q-1}(0,1)$, and thus $x^{\prime} \in F_{0}^{q-1}(0,1)$, where $F_{0}^{q-1}(0,1)=\left\{w \in L(0,1): I^{2-q} w \in A C[0,1]\right.$ and $\left.I^{2-q} w(0)=0\right\}$. Thus, $I^{q-1} g(0)=0$, and hence $x^{\prime}(0)=b_{2}$. Further, for $g \in A C[0,1]$ with $\|g\|=\sup \{|g(t)|, t \in[0,1]\}$, we have

$$
\left|\int_{0}^{t} \frac{(t-s)^{q-2} g(s)}{\Gamma(q-1)} d s\right| \leq\|g\| \frac{t^{q-1}}{\Gamma(q)}<\infty \quad \text { for each } t \in[0,1]
$$


which, together with finiteness of $b_{2}$, implies that $\max \left\{x^{\prime}(t): t \in[0,1]\right\}$ is finite. Next, using the second condition given by (2.2) in (2.4) together with (2.5), we find that

$$
\begin{aligned}
b_{1}= & \left(-\frac{1}{2}+\frac{\xi-\eta}{\zeta}\right) \int_{0}^{1} \frac{(1-s)^{q-1}}{\Gamma(q)} g(s) d s \\
& +\int_{0}^{\frac{1}{2}} \frac{\left(\frac{1}{2}-s\right)^{q-1}}{\Gamma(q)} g(s) d s+\frac{\eta}{\zeta} \int_{0}^{1} \frac{(1-s)^{q-2}}{\Gamma(q-1)} g(s) d s .
\end{aligned}
$$

Substituting the values of $b_{1}$ and $b_{2}$ into (2.4) completes the solution (2.3). Conversely, in view of Lemma 2.3(b), (c) and using the condition that $g \in A C[0,1]$, we have ${ }^{c} D^{q} x(t)=$ $I^{2-q} x^{\prime \prime}(t)=-I^{2-q} D^{2-q} g(t)=-g(t)$, where we have used Remark 2.4 and Lemma 2.3(ii) of [27]. By direct computation it follows that (2.3) satisfies the boundary conditions (2.2). Thus, (2.3) satisfies problem (2.1)-(2.2). This completes the proof.

In view of Lemma 2.5, the solution of problem (1.1) can be expressed in terms of Green's function as

$$
x(t)=\int_{0}^{1}\left[G_{1}(t, s)+G_{2}(t, s)\right] f(s, x(s)) d s,
$$

where

$$
G_{1}(t, s ; q)= \begin{cases}\frac{t(1-s)^{q-1}}{\Gamma(q)} & \text { if } 0 \leq t \leq s \leq 1 \\ -\frac{(t-s)^{q-1}}{\Gamma(q)}+\frac{t(1-s)^{q-1}}{\Gamma(q)} & \text { if } 0 \leq s \leq t \leq 1\end{cases}
$$

and

$$
G_{2}(t, s ; q)= \begin{cases}\left(-\frac{1}{2}+\frac{\xi-\eta}{\zeta}\right) \frac{(1-s)^{q-1}}{\Gamma(q)}+\frac{\eta}{\zeta} \frac{(1-s)^{q-2}}{\Gamma(-1)}+\frac{\left(\frac{1}{2}-s\right)^{q-1}}{\Gamma(q)} & \text { if } 0 \leq s \leq \frac{1}{2} \\ \left(-\frac{1}{2}+\frac{\xi-\eta}{\zeta}\right) \frac{(1-s)^{q-1}}{\Gamma(q)}+\frac{\eta}{\zeta} \frac{(1-s)^{q-2}}{\Gamma(q-1)} & \text { if } \frac{1}{2} \leq s \leq 1\end{cases}
$$

Remark 2.6 For $q=2$, the expressions $G_{1}(t, s ; q)$ and $G_{2}(t, s ; q)$ correspond to the ones associated with a second-order three-point problem discussed in [3].

Let $\mathcal{D}=C([0,1], \mathbb{R})$ denote the Banach space of all continuous functions from $[0,1]$ into $\mathbb{R}$ endowed with the usual norm $\|x\|=\sup \{|x(t)|, t \in[0,1]\}$.

In order to transform problem (1.1) to a fixed point problem, we define the operator $\mathcal{W}: \mathcal{D} \rightarrow \mathcal{D}$ as

$$
\begin{aligned}
(\mathcal{W} x)(t)= & -\int_{0}^{t} \frac{(t-s)^{q-1}}{\Gamma(q)} f(s, x(s)) d s+\left(t-\frac{1}{2}+\frac{\xi-\eta}{\zeta}\right) \int_{0}^{1} \frac{(1-s)^{q-1}}{\Gamma(q)} f(s, x(s)) d s \\
& +\int_{0}^{\frac{1}{2}} \frac{\left(\frac{1}{2}-s\right)^{q-1}}{\Gamma(q)} f(s, x(s)) d s+\frac{\eta}{\zeta} \int_{0}^{1} \frac{(1-s)^{q-2}}{\Gamma(q-1)} f(s, x(s)) d s .
\end{aligned}
$$

In the sequel, we use

$$
\sigma=\frac{2+\xi / \zeta+2^{-q}}{\Gamma(q+1)}+\frac{\eta}{\zeta \Gamma(q)}
$$




\subsection{Existence results}

In this subsection, we present our main results. The first result relies on Banach's contraction mapping principle.

Theorem 2.7 Let $f:[0,1] \times \mathbb{R} \rightarrow \mathbb{R}$ be a continuous function satisfying the Lipschitz condition:

$\left(\mathrm{H}_{1}\right)|f(t, x)-f(t, y)| \leq \ell|x-y|, \forall t \in[0,1], x, y \in \mathbb{R}, \ell>0$.

Then problem (1.1) has a unique solution if $\ell \sigma<1$, where $\sigma$ is given by (2.10).

Proof Let us set $\sup _{t \in[0,1]}|f(t, 0)|=v$ and show that $\mathcal{W} B_{r} \subset B_{r}$, where $B_{r}=\{x \in \mathcal{D}$ : $\|x\| \leq r\}$ with $r>\sigma v(1-\sigma \ell)^{-1}$ and $\mathcal{W}$ is defined by (2.9). For $x \in B_{r}$ and $t \in[0,1]$, using $|f(s, x(s))|=|f(s, x(s))-f(s, 0)+f(s, 0)| \leq \ell r+v$, we get

$$
\begin{aligned}
\|(\mathcal{W} x)\| \leq & \sup _{t \in[0,1]}\left\{\int_{0}^{t} \frac{(t-s)^{q-1}}{\Gamma(q)}|f(s, x(s))-f(s, 0)+f(s, 0)| d s\right. \\
& +\left|t-\frac{1}{2}+\frac{\xi-\eta}{\zeta}\right| \int_{0}^{1} \frac{(1-s)^{q-1}}{\Gamma(q)}|f(s, x(s))-f(s, 0)+f(s, 0)| d s \\
& +\int_{0}^{\frac{1}{2}} \frac{\left(\frac{1}{2}-s\right)^{q-1}}{\Gamma(q)}|f(s, x(s))-f(s, 0)+f(s, 0)| d s \\
& \left.+\frac{\eta}{\zeta} \int_{0}^{1} \frac{(1-s)^{q-2}}{\Gamma(q-1)}|f(s, x(s))-f(s, 0)+f(s, 0)| d s\right\} \\
\leq & (\ell r+v) \sup _{t \in[0,1]}\left\{\int_{0}^{t} \frac{(t-s)^{q-1}}{\Gamma(q)} d s+\left|t-\frac{1}{2}+\frac{\xi-\eta}{\zeta}\right| \int_{0}^{1} \frac{(1-s)^{q-1}}{\Gamma(q)} d s\right. \\
& \left.+\int_{0}^{\frac{1}{2}} \frac{\left(\frac{1}{2}-s\right)^{q-1}}{\Gamma(q)} d s+\frac{\eta}{\zeta} \int_{0}^{1} \frac{(1-s)^{q-2}}{\Gamma(q-1)} d s\right\} \\
\leq & (\ell r+v) \sigma \leq r,
\end{aligned}
$$

which implies that $\mathcal{W} B_{r} \subset B_{r}$, where we have used (2.10). Now, for $x, y \in \mathcal{D}$ and for each $t \in[0,1]$, we obtain

$$
\begin{aligned}
\|\mathcal{W} x-\mathcal{W} y\| \leq & \sup _{t \in[0,1]}\left\{\int_{0}^{t} \frac{(t-s)^{q-1}}{\Gamma(q)}|f(s, x(s))-f(s, y(s))| d s\right. \\
& +\left|t-\frac{1}{2}+\frac{\xi-\eta}{\zeta}\right| \int_{0}^{1} \frac{(1-s)^{q-1}}{\Gamma(q)}|f(s, x(s))-f(s, y(s))| d s \\
& +\int_{0}^{\frac{1}{2}} \frac{\left(\frac{1}{2}-s\right)^{q-1}}{\Gamma(q)}|f(s, x(s))-f(s, y(s))| d s \\
& \left.+\frac{\eta}{\zeta} \int_{0}^{1} \frac{(1-s)^{q-2}}{\Gamma(q-1)}|f(s, x(s))-f(s, y(s))| d s\right\} \\
\leq & \ell \sigma\|x-y\| .
\end{aligned}
$$

Since $\ell \sigma<1$ by the given assumption, the operator $\mathcal{W}$ is a contraction. Thus, by Banach's contraction mapping principle there exists a unique solution for problem (1.1). This completes the proof. 
The next existence result is based on the following fixed point theorem.

Theorem 2.8 Let $X$ be a Banach space. Assume that $T: X \rightarrow X$ is a completely continuous operator and the set

$$
V=\{u \in X \mid u=\mu T u, 0<\mu<1\}
$$

is bounded. Then T has a fixed point in X.

Theorem 2.9 Let $f:[0,1] \times \mathbb{R} \rightarrow \mathbb{R}$ be a continuous function. Suppose that there exists a positive constant $M$ such that $|f(t, x)| \leq M$ for $t \in[0,1], x \in \mathbb{R}$. Then problem (1.1) has at least one solution.

Proof We first show that the operator $\mathcal{W}$ is completely continuous. Obviously, the continuity of the operator $\mathcal{W}$ follows from the continuity of $f$. Let $\mathcal{B} \subset \mathcal{D}$ be a bounded set. By the assumption $|f(t, x)| \leq M$ for $x \in \mathcal{B}$ we have

$$
\begin{aligned}
|(\mathcal{W} x)(t)| \leq & \int_{0}^{t} \frac{(t-s)^{q-1}}{\Gamma(q)}|f(s, x(s))| d s+\left|t-\frac{1}{2}+\frac{\xi-\eta}{\zeta}\right| \int_{0}^{1} \frac{(1-s)^{q-1}}{\Gamma(q)}|f(s, x(s))| d s \\
& +\int_{0}^{\frac{1}{2}} \frac{\left(\frac{1}{2}-s\right)^{q-1}}{\Gamma(q)}|f(s, x(s))| d s+\frac{\eta}{\zeta} \int_{0}^{1} \frac{(1-s)^{q-2}}{\Gamma(q-1)}|f(s, x(s))| d s \\
\leq & M\left[\int_{0}^{t} \frac{(t-s)^{q-1}}{\Gamma(q)} d s+\left|t-\frac{1}{2}+\frac{\xi-\eta}{\zeta}\right| \int_{0}^{1} \frac{(1-s)^{q-1}}{\Gamma(q)} d s\right. \\
& \left.+\int_{0}^{\frac{1}{2}} \frac{\left(\frac{1}{2}-s\right)^{q-1}}{\Gamma(q)} d s+\frac{\eta}{\zeta} \int_{0}^{1} \frac{(1-s)^{q-2}}{\Gamma(q-1)} d s\right] \\
\leq & M \sigma=M_{1},
\end{aligned}
$$

which implies that $\|(\mathcal{W} x)\| \leq M_{1}$. Further, we find that

$$
\begin{aligned}
\left|(\mathcal{W} x)^{\prime}(t)\right| & \leq \int_{0}^{t} \frac{(t-s)^{q-2}}{\Gamma(q-1)}|f(s, x(s))| d s+\int_{0}^{1} \frac{(1-s)^{q-1}}{\Gamma(q)}|f(s, x(s))| d s \\
& \leq M\left[\int_{0}^{t} \frac{(t-s)^{q-2}}{\Gamma(q-1)} d s+\int_{0}^{1} \frac{(1-s)^{q-1}}{\Gamma(q)} d s\right] \\
& \leq \frac{M(q+1)}{\Gamma(q+1)}=M_{2} .
\end{aligned}
$$

Hence, for $t_{1}, t_{2} \in[0,1]$, we have

$$
\left|(\mathcal{W} x)\left(t_{2}\right)-(\mathcal{W} x)\left(t_{1}\right)\right| \leq \int_{t_{1}}^{t_{2}}\left|(\mathcal{W} x)^{\prime}(s)\right| d s \leq M_{2}\left(t_{2}-t_{1}\right)
$$

This implies that $\mathcal{W}$ is equicontinuous on $[0,1]$. Thus, by the Arzelà-Ascoli theorem the operator $\mathcal{W}: \mathcal{D} \rightarrow \mathcal{D}$ is completely continuous.

Next, we consider the set

$$
\mathcal{A}=\{x \in \mathcal{D} \mid x=\nu \mathcal{W} x, 0<\nu<1\}
$$


and show that the set $\mathcal{A}$ is bounded. Let $x \in \mathcal{A}$; then $x=\nu \mathcal{W} x, 0<v<1$. For any $t \in[0,1]$, we have

$$
\begin{aligned}
x(t)= & -\int_{0}^{t} \frac{(t-s)^{q-1}}{\Gamma(q)} f(s, x(s)) d s+\left(t-\frac{1}{2}+\frac{\xi-\eta}{\zeta}\right) \int_{0}^{1} \frac{(1-s)^{q-1}}{\Gamma(q)} f(s, x(s)) d s \\
& +\int_{0}^{\frac{1}{2}} \frac{\left(\frac{1}{2}-s\right)^{q-1}}{\Gamma(q)} f(s, x(s)) d s+\frac{\eta}{\zeta} \int_{0}^{1} \frac{(1-s)^{q-2}}{\Gamma(q-1)} f(s, x(s)) d s
\end{aligned}
$$

and $|x(t)|=v|(\mathcal{W} x)(t)| \leq|(\mathcal{W} x)(t)| \leq M \sigma=M_{1}$ for any $t \in[0,1]$. So, the set $\mathcal{A}$ is bounded. Thus, the conclusion of Theorem 2.8 applies, and the operator $\mathcal{W}$ has at least one fixed point. This, in turn, implies that problem (1.1) has at least one solution on $[0,1]$.

Now we show the existence of solutions for problem (1.1) by means of Leray-Schauder degree theory.

Theorem 2.10 Let $f:[0,1] \times \mathbb{R} \rightarrow \mathbb{R}$ be a continuous function. Suppose that there exist constants $0 \leq \varrho<1 / \sigma$ and $N>0$ such that $|f(t, x)| \leq \varrho|x|+N$ for all $t \in[0,1], x \in \mathcal{D}$. Then problem (1.1) has at least one solution.

Proof Define a ball $B_{R} \subset \mathcal{D}$ with radius $R>0$ by

$$
B_{R}=\left\{x \in \mathcal{D}: \max _{t \in[0,1]}|x(t)|<R\right\},
$$

where $R$ will be fixed later. Then, it is enough to show that the operator $\mathcal{W}: \bar{B}_{R} \rightarrow \mathcal{D}$ (given by (2.9)) is such that

$$
x \neq \lambda \mathcal{W} x, \quad \forall x \in \partial B_{R} \text { and } \forall \lambda \in[0,1] .
$$

Now we set

$$
\mathcal{K}(\lambda, x)=\lambda \mathcal{W} x, \quad x \in C(\mathbb{R}), \lambda \in[0,1]
$$

Then, by the Arzelà-Ascoli theorem, $\omega_{\lambda}(x)=x-\mathcal{K}(\lambda, x)=x-\lambda \mathcal{W} x$ is completely continuous. If the condition (2.14) holds, then the following Leray-Schauder degrees are well defined, and by the homotopy invariance of topological degree we have that

$$
\begin{aligned}
\operatorname{deg}\left(\omega_{\lambda}, B_{R}, 0\right) & =\operatorname{deg}\left(I-\lambda \mathcal{W}, B_{R}, 0\right)=\operatorname{deg}\left(\omega_{1}, B_{R}, 0\right) \\
& =\operatorname{deg}\left(\omega_{0}, B_{R}, 0\right)=\operatorname{deg}\left(I, B_{R}, 0\right)=1 \neq 0, \quad 0 \in B_{R},
\end{aligned}
$$

where $I$ denotes the unit operator. By the nonzero property of Leray-Schauder degree, $\omega_{1}(t)=x-\lambda \mathcal{W} x=0$ for at least one $x \in B_{R}$. In order to justify condition (2.14), we assume that $x=\lambda \mathcal{W} x$ for some $\lambda \in[0,1]$ and for all $t \in[0,1]$, so that

$$
\begin{aligned}
|x(t)| & =|\lambda \mathcal{W} x(t)| \\
& \leq \int_{0}^{t} \frac{(t-s)^{q-1}}{\Gamma(q)}|f(s, x(s))| d s+\left(t-\frac{1}{2}+\frac{\xi-\eta}{\zeta}\right) \int_{0}^{1} \frac{(1-s)^{q-1}}{\Gamma(q)}|f(s, x(s))| d s
\end{aligned}
$$




$$
\begin{aligned}
& +\int_{0}^{\frac{1}{2}} \frac{\left(\frac{1}{2}-s\right)^{q-1}}{\Gamma(q)}|f(s, x(s))| d s+\frac{\eta}{\zeta} \int_{0}^{1} \frac{(1-s)^{q-2}}{\Gamma(q-1)}|f(s, x(s))| d s \\
\leq & (\varrho\|x\|+N)\left\{\int_{0}^{t} \frac{(t-s)^{q-1}}{\Gamma(q)} d s+\left|t-\frac{1}{2}+\frac{\xi-\eta}{\zeta}\right| \int_{0}^{1} \frac{(1-s)^{q-1}}{\Gamma(q)} d s\right. \\
& \left.+\int_{0}^{\frac{1}{2}} \frac{\left(\frac{1}{2}-s\right)^{q-1}}{\Gamma(q)} d s+\frac{\eta}{\zeta} \int_{0}^{1} \frac{(1-s)^{q-2}}{\Gamma(q-1)} d s\right\}
\end{aligned}
$$

which, on taking the norm over the interval $[0,1]$, yields

$$
\|x\| \leq(\varrho\|x\|+N) \sigma
$$

where $\sigma$ is given by (2.10). In consequence we have

$$
\|x\| \leq \frac{\sigma N}{1-\varrho \sigma}
$$

Letting $R=\sigma N /(1-\varrho \sigma)+1,(2.14)$ holds. This completes the proof.

The next result is based on Krasnoselskii's fixed point theorem [28].

Theorem 2.11 Let $f:[0,1] \times \mathbb{R} \rightarrow \mathbb{R}$ be a continuous function satisfying $\left(\mathrm{H}_{1}\right)$ and

$\left(\mathrm{H}_{2}\right)|f(t, x)| \leq \mu(t), \forall(t, x) \in[0,1] \times \mathbb{R}$, and $\mu \in C\left([0,1], \mathbb{R}^{+}\right)$.

Then problem (1.1) has at least one solution on $[0,1]$ if

$$
\frac{1+\xi / \zeta+2^{-q}}{\Gamma(q+1)}+\frac{\eta}{\zeta \Gamma(q)}<1
$$

Proof For $r \geq\|\mu\| \rho$, let us define a closed set (ball) $B_{r}=\{x \in \mathcal{D}:\|x\| \leq r\}$ and introduce the operators $\mathcal{W}_{1}$ and $\mathcal{W}_{2}$ on $B_{r}$ defined as

$$
\begin{aligned}
\left(\mathcal{W}_{1} x\right)(t)= & -\int_{0}^{t} \frac{(t-s)^{q-1}}{\Gamma(q)} f(s, x(s)) d s \\
\left(\mathcal{W}_{2} x\right)(t)= & \left(t-\frac{1}{2}+\frac{\xi-\eta}{\zeta}\right) \int_{0}^{1} \frac{(1-s)^{q-1}}{\Gamma(q)} f(s, x(s)) d s \\
& +\int_{0}^{\frac{1}{2}} \frac{\left(\frac{1}{2}-s\right)^{q-1}}{\Gamma(q)} f(s, x(s)) d s+\frac{\eta}{\zeta} \int_{0}^{1} \frac{(1-s)^{q-2}}{\Gamma(q-1)} f(s, x(s)) d s .
\end{aligned}
$$

For $x, y \in B_{r}$, it is easy to show that $\left\|\left(\mathcal{W}_{1} x\right)+\left(\mathcal{W}_{2} y\right)\right\| \leq\|\mu\| \sigma \leq r$, where $\sigma$ is given by (2.10). This implies that $\mathcal{W}_{1} x+\mathcal{W}_{2} y \in B_{r}$.

In view of condition (2.15), the operator $\mathcal{W}_{2}$ is a contraction. The continuity of the operator $\mathcal{W}_{1}$ follows from that of $f$. Also, $\left\|\mathcal{W}_{1} x\right\| \leq\|\mu\| / \Gamma(q+1)$ implies that $\mathcal{W}_{1}$ is uniformly bounded on $B_{r}$. Furthermore, with $\sup _{(t, x) \in[0,1] \times B_{r}}|f(t, x)|=f_{b}<\infty$ and $t_{1}, t_{2} \in(0,1]$, we have

$$
\left\|\left(\mathcal{W}_{1} x\right)\left(t_{2}\right)-\left(\mathcal{W}_{1} x\right)\left(t_{1}\right)\right\| \leq \frac{f_{b}}{\Gamma(q+1)}\left(\left|t_{2}^{q}-t_{1}^{q}\right|+2\left(\left|t_{2}-t_{1}\right|\right)^{q}\right) \rightarrow 0
$$


independent of $x$ as $t_{1} \rightarrow t_{2}$. This shows that $\mathcal{W}_{1}$ is relatively compact on $B_{r}$. Hence, we infer by the Arzelà-Ascoli theorem that $\mathcal{W}_{1}$ is compact on $B_{r}$. Thus, all the conditions of Krasnoselskii's fixed point theorem are satisfied. Hence, problem (1.1) has at least one solution on $[0,1]$. This completes the proof.

Finally, we apply the Leray-Schauder nonlinear alternative to show the existence of solutions for problem (1.1).

Lemma 2.12 (Nonlinear alternative for single-valued maps [29]) Let E be a Banach space, $E_{1}$ a closed, convex subset of $E, V$ an open subset of $E_{1}$, and $0 \in V$. Suppose that $\mathcal{U}: \bar{V} \rightarrow E_{1}$ is a continuous compact (that is, $\mathcal{U}(\bar{V})$ is a relatively compact subset of $E_{1}$ ) map. Then either

(i) $\mathcal{U}$ has a fixed point in $\bar{V}$, or

(ii) there are an $x \in \partial V$ (the boundary of $V$ in $E_{1}$ ) and $\kappa \in(0,1)$ with $x=\kappa \mathcal{U}(x)$.

Theorem 2.13 Let $f:[0,1] \times \mathbb{R} \rightarrow \mathbb{R}$ be a continuous function. Further, assume that

$\left(\mathrm{H}_{3}\right)$ there exist a function $p \in \mathcal{C}\left([0,1], \mathbb{R}^{+}\right)$and a nondecreasing function $\psi: \mathbb{R}^{+} \rightarrow \mathbb{R}^{+}$such that $|f(t, x)| \leq p(t) \psi(\|x\|), \forall(t, x) \in[0,1] \times \mathbb{R} ;$

$\left(\mathrm{H}_{4}\right)$ there exists a constant $M>0$ such that

$$
\frac{M}{\psi(M)\|p\| \sigma}>1
$$

where $\sigma$ is given by (2.10).

Then problem (1.1) has at least one solution on $[0,1]$.

Proof As a first step, we show that the operator $\mathcal{W}: \mathcal{D} \rightarrow \mathcal{D}$ defined by (2.9) maps bounded sets into bounded sets in $\mathcal{D}$. For a positive number $r$, let $B_{r}=\{x \in \mathcal{D}:\|x\| \leq r\}$ be a bounded set in $\mathcal{D}$. Then, for $x \in B_{r}$ together with $\left(\mathrm{H}_{3}\right)$ and $\left(\mathrm{H}_{4}\right)$, we obtain

$$
\begin{aligned}
|(\mathcal{W} x)(t)| \leq & \int_{0}^{t} \frac{(t-s)^{q-1}}{\Gamma(q)} p(s) \psi(\|x\|) d s+\left|t-\frac{1}{2}+\frac{\xi-\eta}{\zeta}\right| \int_{0}^{1} \frac{(1-s)^{q-1}}{\Gamma(q)} p(s) \psi(\|x\|) d s \\
& +\int_{0}^{\frac{1}{2}} \frac{\left(\frac{1}{2}-s\right)^{q-1}}{\Gamma(q)} p(s) \psi(\|x\|) d s+\frac{\eta}{\zeta} \int_{0}^{1} \frac{(1-s)^{q-2}}{\Gamma(q-1)} p(s) \psi(\|x\|) d s \\
\leq & \|p\| \sigma \psi(r)<r .
\end{aligned}
$$

Next, we will show that $\mathcal{W}$ maps bounded sets into equicontinuous sets of $\mathcal{D}$. Let $t_{1}, t_{2} \in$ $[0,1]$ and $x \in B_{r}$. Then

$$
\begin{aligned}
& \left|(\mathcal{W} x)\left(t_{2}\right)-(\mathcal{W} x)\left(t_{1}\right)\right| \\
& =\mid-\int_{0}^{t_{2}} \frac{\left(t_{2}-s\right)^{q-1}}{\Gamma(q)} f(s, x(s)) d s+\int_{0}^{t_{1}} \frac{\left(t_{1}-s\right)^{q-1}}{\Gamma(q)} f(s, x(s)) d s \\
& \quad+\left(t_{2}-t_{1}\right) \int_{0}^{1} \frac{(1-s)^{q-1}}{\Gamma(q)} f(s, x(s)) d s \mid \\
& \quad \leq \frac{\psi(r)\|p\|}{\Gamma(q+1)}\left(\left|t_{2}^{q}-t_{1}^{q}\right|+2\left(\left|t_{2}-t_{1}\right|\right)^{q}+\left|t_{2}-t_{1}\right|\right) .
\end{aligned}
$$


Clearly, the right-hand side of this inequality tends to zero independently of $x \in B_{r}$ as $t_{2} \rightarrow t_{1}$. Thus, by the Arzelà-Ascoli theorem, the operator $\mathcal{W}$ is completely continuous.

Let $x$ be a solution for the given problem. Then, for $\lambda \in(0,1)$, as before, we obtain

$$
\|x\|=\sup _{t \in[0,1]}|\lambda(\mathcal{W} x)(t)| \leq \psi(\|x\|)\|p\| \sigma
$$

In view of $\left(\mathrm{H}_{4}\right)$, there exists $M$ such that $\|x\| \neq M$. Let us choose $\mathcal{P}=\{x \in \mathcal{D}:\|x\|<M+1\}$. Notice that the operator $\mathcal{W}: \overline{\mathcal{P}} \rightarrow \mathcal{D}$ is continuous and completely continuous. From the choice of $\mathcal{P}$, there is no $x \in \partial \mathcal{P}$ such that $x=\lambda \mathcal{W}(x)$ for some $\lambda \in(0,1)$. Consequently, the conclusion of Lemma 2.12 applies, and hence the operator $\mathcal{W}$ has a fixed point $x \in \overline{\mathcal{P}}$, which is a solution of problem (1.1). This completes the proof.

\subsection{Examples}

Consider the three-point boundary value problem of nonlinear fractional differential equations

$$
\begin{aligned}
& { }^{c} D^{7 / 4} x(t)+f(t, x(t))=0, \quad t \in[0,1], 1<q \leq 2, \\
& x(0)=x(1), \quad \frac{3}{2} x^{\prime}(0)-\frac{1}{2} x^{\prime}(1)=\frac{1}{2} x(1 / 2),
\end{aligned}
$$

where $q=7 / 4, \xi=3 / 2, \eta=1 / 2, \zeta=1 / 2$. Clearly, $\xi / \zeta>(1 / 2+\eta / \zeta)$ and

$$
\sigma=\frac{2+\xi / \zeta+2^{-q}}{\Gamma(q+1)}+\frac{\eta}{\zeta \Gamma(q)} \simeq 4.381671
$$

(a) In (2.16), let us choose any continuous function $f(t, x(t))$ such that $|f(t, x)-f(t, y)| \leq$ $\ell|x-y|, \forall t \in[0,1], x, y \in \mathbb{R}$ with $\ell<1 / \sigma$, where $\sigma$ is given by (2.18). Then problem (2.16)(2.17) has a unique solution on $[0,1]$ by Theorem 2.7. For instance, for $f(t, x)=e^{-t(1-t)}(|x|+$ $\left.\tan ^{-1} x+1\right) / 10$, it is clear that $\ell=1 / 5<1 / \sigma \simeq 0.2282234$.

(b) We take $f(t, x)$ in (2.16) such that $|f(t, x)| \leq p(t) \psi(\|x\|)$ with $\|p\|=p_{1}$ and $\psi(\|x\|)=$ $1+a\|x\|$, where $p_{1}$ and $a$ are appropriate real numbers. Then, by condition $\left(\mathrm{H}_{4}\right)$ we find that

$$
M>\frac{p_{1} \sigma}{\left(1-p_{1} a \sigma\right)}
$$

where $\sigma$ is given by (2.18). Then the conclusion of Theorem 2.13 applies, and hence problem (2.16)-(2.17) with the given value of $f(t, x)$ has at least one solution on $[0,1]$. In particular, by taking $f(t, x)=\frac{4}{25}\left(1+t-t^{2}\right)\left[2\left(\cos ^{2}(t(1-t))+1\right) \sin |x| / 5+e^{-t(1-t)}\right]$ we find that $|f(t, x)| \leq \frac{4}{25}\left(1+t-t^{2}\right)(1+4\|x\| / 5), p_{1}=1 / 5, a=4 / 5$, and $M>2.931544$.

\section{Multivalued case}

In this section, we consider the multivalued variant of problem (1.1)

$$
\begin{aligned}
& { }^{c} D^{q} x(t) \in-F(t, x(t)), \quad t \in[0,1], 1<q \leq 2, \\
& x(0)=x(1), \quad \xi x^{\prime}(0)-\eta x^{\prime}(1)=\zeta x(1 / 2),
\end{aligned}
$$


where $F:[0,1] \times \mathbb{R} \rightarrow \mathcal{P}(\mathbb{R})$ is a compact-valued map, and $\mathcal{P}(\mathbb{R})$ is the family of all nonempty subsets of $\mathbb{R}$. We discuss the existence of solutions for problem (3.1) when the multivalued map $F(t, x(t))$ is convex or nonconvex set-valued by using the nonlinear alternative of Leray-Schauder type and a fixed point theorem for generalized contractions due to Wegrzyk.

\subsection{Some definitions}

This subsection is devoted to some basic definitions on multivalued maps [30, 31].

Definition 3.1 Let $(\mathcal{X},\|\cdot\|)$ be a normed space, and let $P(\mathcal{X})=\{\mathcal{Y} \subseteq \mathcal{X}: \mathcal{Y} \neq \emptyset\}, P_{b}(\mathcal{X})=$ $\{\mathcal{Y} \in \mathcal{P}(\mathcal{X}): \mathcal{Y}$ is bounded $\}$, and $P_{c l}(\mathcal{X})=\{\mathcal{Y} \in \mathcal{P}(\mathcal{X}): \mathcal{Y}$ is closed $\}$. Then a multivalued map $\mathcal{H}: \mathcal{X} \rightarrow \mathcal{P}(\mathcal{X})$

(a) is convex (closed) valued if $\mathcal{H}(x)$ is convex (closed) for all $x \in \mathcal{X}$;

(b) is bounded on bounded sets if $\mathcal{H}(\mathbb{B})=\cup_{x \in \mathbb{B}} \mathcal{H}(x)$ is bounded in $\mathcal{X}$ for all $\mathbb{B} \in P_{b}(\mathcal{X})$ (i.e., $\sup _{x \in \mathbb{B}}\{\sup \{|y|: y \in \mathcal{H}(x)\}\}<\infty$ );

(c) is called upper semicontinuous (u.s.c.) on $\mathcal{X}$ if for each $x_{0} \in \mathcal{X}$, the set $\mathcal{H}\left(x_{0}\right)$ is a nonempty closed subset of $\mathcal{X}$ and if for each open set $N$ of $\mathcal{X}$ containing $\mathcal{H}\left(x_{0}\right)$, there exists an open neighborhood $\mathcal{N}_{0}$ of $x_{0}$ such that $\mathcal{H}\left(\mathcal{N}_{0}\right) \subseteq N$;

(d) is said to be completely continuous if $\mathcal{H}(\mathbb{B})$ is relatively compact for every $\mathbb{B} \in P_{b}(\mathcal{X})$;

(e) has a fixed point if there is $x \in \mathcal{X}$ such that $x \in \mathcal{H}(x)$.

The fixed point set of a multivalued operator $\mathcal{H}$ will be denoted by Fix $\mathcal{H}$.

Remark 3.2 It is known that if a multivalued map $\mathcal{H}$ is completely continuous with nonempty compact values, then $G$ is u.s.c. if and only if $\mathcal{H}$ has a closed graph, that is, $x_{n} \rightarrow x_{*}, y_{n} \rightarrow y_{*}, y_{n} \in \mathcal{H}\left(x_{n}\right)$ imply $y_{*} \in \mathcal{H}\left(x_{*}\right)$.

Definition 3.3 A multivalued map $\mathcal{H}:[0 ; 1] \rightarrow P_{c l}(\mathbb{R})$ is said to be measurable if for every $y \in \mathbb{R}$, the function $t \longmapsto d(y, \mathcal{H}(t))=\inf \{\|y-z\|: z \in \mathcal{H}(t)\}$ is measurable.

Definition 3.4 A multivalued map $F:[0,1] \times \mathbb{R} \rightarrow \mathcal{P}(\mathbb{R})$ is said to be Carathéodory if (a) $t \longmapsto F(t, x)$ is measurable for each $x \in \mathbb{R}$ and (ii) $x \longmapsto F(t, x)$ is upper semicontinuous for almost all $t \in[0,1]$. Further, a Carathéodory function $F$ is called $L^{1}$-Carathéodory if

(iii) for each $a>0$, there exists $\varphi_{a} \in L^{1}\left([0,1], \mathbb{R}^{+}\right)$such that

$$
\|F(t, x)\|=\sup \{|v|: v \in F(t, x)\} \leq \varphi_{a}(t)
$$

for all $\|x\| \leq a$ and for a.e. $t \in[0,1]$.

For each $y \in C([0,1], \mathbb{R})$, define the set of selections of $F$ by

$$
S_{F, y}:=\left\{v \in L^{1}([0,1], \mathbb{R}): v(t) \in F(t, y(t)) \text { for a.e. } t \in[0,1]\right\} .
$$

We denote by $C([0,1])$ the Banach space of continuous functions from $[0,1]$ into $\mathbb{R}$ with the norm $\|x\|_{\infty}=\sup _{t \in[0,1]}|x(t)|$. Let $L^{1}([0,1], \mathbb{R})$ be the Banach space of Lebesguemeasurable and integrable functions $x:[0,1] \rightarrow \mathbb{R}$ with norm $\|x\|_{L^{1}}=\int_{0}^{1}|x(t)| d t$.

Definition 3.5 A function $x \in A C^{2}([0,1], \mathbb{R})$ is a solution of problem (3.1) if there exists a function $f \in L^{1}([0,1], \mathbb{R})$ such that $f(t) \in F(t, x(t))$ a.e. on $[0,1]$ and 


$$
\begin{aligned}
x(t)= & -\int_{0}^{t} \frac{(t-s)^{q-1}}{\Gamma(q)} f(s) d s+\left(t-\frac{1}{2}+\frac{\xi-\eta}{\zeta}\right) \int_{0}^{1} \frac{(1-s)^{q-1}}{\Gamma(q)} f(s) d s \\
& +\int_{0}^{\frac{1}{2}} \frac{\left(\frac{1}{2}-s\right)^{q-1}}{\Gamma(q)} f(s) d s+\frac{\eta}{\zeta} \int_{0}^{1} \frac{(1-s)^{q-2}}{\Gamma(q-1)} f(s) d s .
\end{aligned}
$$

\subsection{Convex set-valued case}

In our first result, we need the following lemmas.

Lemma 3.6 (Nonlinear alternative of Leray-Schauder type [29]) Let $C$ be a convex set in a normed space, and $U \subset C$ be open with $0 \in U$. Then each compact and upper semicontinuous mapping $\Phi: \bar{U} \rightarrow P(C)$ with compact convex values that is fixed point free on $\partial U$ has at least one of the following two properties: (a) $\Phi$ has a fixed point; (b) there exist $x \in \partial U$ and $\lambda \in(0,1)$ such that $x \in \lambda \Phi(x)$.

Lemma 3.7 (Arzelà-Ascoli theorem $[29])$ A subset of $C([0,1], \mathbb{R})$ is relatively compact if and only if it is bounded and equicontinuous.

Lemma 3.8 ([32]) Let $X$ be a Banach space. Let $F:[0 ; 1] \times \mathbb{R} \rightarrow P_{c p, c}(X)$ be an $L^{1}$ Carathéodory multivalued map, and let $\Theta$ be a linear continuous mapping from $L^{1}([0,1], X)$ to $C([0,1], X)$. Then the operator

$$
\Theta \circ S_{F}: C([0,1], X) \rightarrow P_{c p, c}(C([0,1], X)), \quad x \mapsto\left(\Theta \circ S_{F}\right)(x)=\Theta\left(S_{F, x}\right),
$$

is a closed graph operator in $C([0,1], X) \times C([0,1], X)$, where $P_{c p, c}(X)=\{Y \in \mathcal{P}(X)$ : $Y$ is compact and convex\}.

Theorem 3.9 Assume that

$\left(\mathrm{H}_{1}\right) \quad F:[0,1] \times \mathbb{R} \rightarrow \mathcal{P}(\mathbb{R})$ is $L^{1}$-Carathéodory and has compact and convex values;

$\left(\mathrm{H}_{2}\right)$ there exist a continuous nondecreasing function $\psi:[0, \infty) \rightarrow(0, \infty)$ and a function $p \in C\left([0,1], \mathbb{R}_{+}\right)$such that

$$
\|F(t, x)\|_{\mathcal{P}}:=\sup \{|y|: y \in F(t, x)\} \leq p(t) \psi(|x|) \quad \text { for all }(t, x) \in[0,1] \times \mathbb{R}
$$

$\left(\mathrm{H}_{3}\right)$ there exists a number $M>0$ such that

$$
\frac{M}{\psi(M)\|p\|_{\infty}}\left[\frac{\left(2+\xi / \zeta+2^{-q}\right)}{\Gamma(q+1)}+\frac{\eta}{\zeta \Gamma(q)}\right]^{-1}>1
$$

Then the boundary value problem (3.1) has at least one solution on $[0,1]$.

Proof Define the operator $\Omega: C([0,1], \mathbb{R}) \rightarrow \mathcal{P}(C([0,1], \mathbb{R}))$ by

$$
\begin{aligned}
\Omega(x)= & \left\{\mathfrak{h} \in C([0,1], \mathbb{R}): \mathfrak{h}(t)=-\int_{0}^{t} \frac{(t-s)^{q-1}}{\Gamma(q)} f(s) d s\right. \\
& +\left(t-\frac{1}{2}+\frac{\xi-\eta}{\zeta}\right) \int_{0}^{1} \frac{(1-s)^{q-1}}{\Gamma(q)} f(s) d s \\
& \left.+\int_{0}^{\frac{1}{2}} \frac{\left(\frac{1}{2}-s\right)^{q-1}}{\Gamma(q)} f(s) d s+\frac{\eta}{\zeta} \int_{0}^{1} \frac{(1-s)^{q-2}}{\Gamma(q-1)} f(s) d s, f \in S_{F, x}\right\} .
\end{aligned}
$$


We will show that the operator $\Omega$ satisfies the assumptions of Lemma 3.6. The proof consists of several steps. In the first step, we show that $\Omega(x)$ is convex for each $x \in C([0,1], \mathbb{R})$. For that, let $\mathfrak{h}_{1}, \mathfrak{h}_{2} \in \Omega(x)$. Then there exist $f_{1}, f_{2} \in S_{F, x}$ such that for each $t \in[0,1]$, we have

$$
\begin{aligned}
\mathfrak{h}_{i}(t)= & -\int_{0}^{t} \frac{(t-s)^{q-1}}{\Gamma(q)} f_{i}(s) d s+\left(t-\frac{1}{2}+\frac{\xi-\eta}{\zeta}\right) \int_{0}^{1} \frac{(1-s)^{q-1}}{\Gamma(q)} f_{i}(s) d s \\
& +\int_{0}^{\frac{1}{2}} \frac{\left(\frac{1}{2}-s\right)^{q-1}}{\Gamma(q)} f_{i}(s) d s+\frac{\eta}{\zeta} \int_{0}^{1} \frac{(1-s)^{q-2}}{\Gamma(q-1)} f_{i}(s) d s, \quad i=1,2 .
\end{aligned}
$$

Let $0 \leq \lambda \leq 1$. Then, for each $t \in[0,1]$, we have

$$
\begin{aligned}
{\left[\lambda \mathfrak{h}_{1}+(1-\lambda) \mathfrak{h}_{2}\right](t)=} & -\int_{0}^{t} \frac{(t-s)^{q-1}}{\Gamma(q)}\left[\lambda f_{1}(s)+(1-\lambda) f_{2}(s)\right] d s \\
& +\left(t-\frac{1}{2}+\frac{\xi-\eta}{\zeta}\right) \int_{0}^{1} \frac{(1-s)^{q-1}}{\Gamma(q)}\left[\lambda f_{1}(s)+(1-\lambda) f_{2}(s)\right] d s \\
& +\int_{0}^{\frac{1}{2}} \frac{\left(\frac{1}{2}-s\right)^{q-1}}{\Gamma(q)}\left[\lambda f_{1}(s)+(1-\lambda) f_{2}(s)\right] d s \\
& +\frac{\eta}{\zeta} \int_{0}^{1} \frac{(1-s)^{q-2}}{\Gamma(q-1)}\left[\lambda f_{1}(s)+(1-\lambda) f_{2}(s)\right] d s .
\end{aligned}
$$

Since $S_{F, x}$ is convex ( $F$ has convex values), it follows that $\lambda \mathfrak{h}_{1}+(1-\lambda) \mathfrak{h}_{2} \in \Omega(x)$.

Next, we show that $\Omega$ maps bounded sets into bounded sets in $C([0,1], \mathbb{R})$. For a positive number $r_{1}$, let $B_{r_{1}}=\left\{x \in C([0,1], \mathbb{R}):\|x\|_{\infty} \leq r_{1}\right\}$ be a bounded set in $C([0,1], \mathbb{R})$. Then, for each $\mathfrak{h} \in \Omega(x), x \in B_{r_{1}}$, there exists $f \in S_{F, x}$ such that

$$
\begin{aligned}
\mathfrak{h}(t)= & -\int_{0}^{t} \frac{(t-s)^{q-1}}{\Gamma(q)} f(s) d s+\left(t-\frac{1}{2}+\frac{\xi-\eta}{\zeta}\right) \int_{0}^{1} \frac{(1-s)^{q-1}}{\Gamma(q)} f(s) d s \\
& +\int_{0}^{\frac{1}{2}} \frac{\left(\frac{1}{2}-s\right)^{q-1}}{\Gamma(q)} f(s) d s+\frac{\eta}{\zeta} \int_{0}^{1} \frac{(1-s)^{q-2}}{\Gamma(q-1)} f(s) d s,
\end{aligned}
$$

and, as in Section 3, we can get

$$
\|\mathfrak{h}\|_{\infty} \leq\left[\frac{\left(2+\xi / \zeta+2^{-q}\right)}{\Gamma(q+1)}+\frac{\eta}{\zeta \Gamma(q)}\right]\|p\|_{\infty} \psi\left(r_{1}\right) .
$$

Now we show that $\Omega$ maps bounded sets into equicontinuous sets in $C([0,1], \mathbb{R})$. Let $\tau_{1}, \tau_{2} \in[0,1]$ with $\tau_{1}<\tau_{2}$ and $x \in B_{r_{1}}$, where $B_{r_{1}}$ is a bounded set in $C([0,1], \mathbb{R})$. For each $\mathfrak{h} \in \Omega(x)$, we obtain

$$
\left|\mathfrak{h}\left(\tau_{2}\right)-\mathfrak{h}\left(\tau_{1}\right)\right| \leq \frac{\psi\left(r_{1}\right)\|p\|_{\infty}}{\Gamma(q+1)}\left(\left|\tau_{2}^{q}-\tau_{1}^{q}\right|+2\left(\left|\tau_{2}-\tau_{1}\right|\right)^{q}+\left|\tau_{2}-\tau_{1}\right|\right)
$$

Obviously the right-hand side of this inequality tends to zero independently of $x \in B_{r_{1}}$ as $\tau_{2}-\tau_{1} \rightarrow 0$. In view of the above three steps, it follows by Lemma 3.7 that $\Omega$ is completely continuous.

Now we show that $\Omega$ has a closed graph. Let $x_{n} \rightarrow x_{*}, \mathfrak{h}_{n} \in \Omega\left(x_{n}\right)$, and $\mathfrak{h}_{n} \rightarrow \mathfrak{h}_{*}$. Then we need to show that $\mathfrak{h}_{*} \in \Omega\left(x_{*}\right)$. Associated with $\mathfrak{h}_{n} \in \Omega\left(x_{n}\right)$, there exists $f_{n} \in S_{F, x_{n}}$ such 
that for each $t \in[0,1]$,

$$
\begin{aligned}
\mathfrak{h}_{n}(t)= & -\int_{0}^{t} \frac{(t-s)^{q-1}}{\Gamma(q)} f_{n}(s) d s+\left(t-\frac{1}{2}+\frac{\xi-\eta}{\zeta}\right) \int_{0}^{1} \frac{(1-s)^{q-1}}{\Gamma(q)} f_{n}(s) d s \\
& +\int_{0}^{\frac{1}{2}} \frac{\left(\frac{1}{2}-s\right)^{q-1}}{\Gamma(q)} f_{n}(s) d s+\frac{\eta}{\zeta} \int_{0}^{1} \frac{(1-s)^{q-2}}{\Gamma(q-1)} f_{n}(s) d s .
\end{aligned}
$$

Thus, we have to show that there exists $f_{*} \in S_{F, x_{*}}$ such that for each $t \in[0,1]$,

$$
\begin{aligned}
\mathfrak{h}_{*}(t)= & -\int_{0}^{t} \frac{(t-s)^{q-1}}{\Gamma(q)} f_{*}(s) d s+\left(t-\frac{1}{2}+\frac{\xi-\eta}{\zeta}\right) \int_{0}^{1} \frac{(1-s)^{q-1}}{\Gamma(q)} f_{*}(s) d s \\
& +\int_{0}^{\frac{1}{2}} \frac{\left(\frac{1}{2}-s\right)^{q-1}}{\Gamma(q)} f_{*}(s) d s+\frac{\eta}{\zeta} \int_{0}^{1} \frac{(1-s)^{q-2}}{\Gamma(q-1)} f_{*}(s) d s .
\end{aligned}
$$

Consider the continuous linear operator $\Theta: L^{1}([0,1], \mathbb{R}) \rightarrow C([0,1], \mathbb{R})$ given by

$$
\begin{aligned}
f \mapsto \Theta(f)(t)= & -\int_{0}^{t} \frac{(t-s)^{q-1}}{\Gamma(q)} f(s) d s+\left(t-\frac{1}{2}+\frac{\xi-\eta}{\zeta}\right) \int_{0}^{1} \frac{(1-s)^{q-1}}{\Gamma(q)} f(s) d s \\
& +\int_{0}^{\frac{1}{2}} \frac{\left(\frac{1}{2}-s\right)^{q-1}}{\Gamma(q)} f(s) d s+\frac{\eta}{\zeta} \int_{0}^{1} \frac{(1-s)^{q-2}}{\Gamma(q-1)} f(s) d s .
\end{aligned}
$$

Observe that

$$
\begin{aligned}
\left\|\mathfrak{h}_{n}(t)-\mathfrak{h}_{*}(t)\right\|= & \|-\int_{0}^{t} \frac{(t-s)^{q-1}}{\Gamma(q)}\left(f_{n}(s)-f_{*}(s)\right) d s \\
& +\left(t-\frac{1}{2}+\frac{\xi-\eta}{\zeta}\right) \int_{0}^{1} \frac{(1-s)^{q-1}}{\Gamma(q)}\left(f_{n}(s)-f_{*}(s)\right) d s \\
& +\int_{0}^{\frac{1}{2}} \frac{\left(\frac{1}{2}-s\right)^{q-1}}{\Gamma(q)}\left(f_{n}(s)-f_{*}(s)\right) d s \\
& +\frac{\eta}{\zeta} \int_{0}^{1} \frac{(1-s)^{q-2}}{\Gamma(q-1)}\left(f_{n}(s)-f_{*}(s)\right) d s \| \rightarrow 0 \quad \text { as } n \rightarrow \infty .
\end{aligned}
$$

Thus, it follows by Lemma 3.8 that $\Theta \circ S_{F}$ is a closed graph operator. Further, we have $\mathfrak{h}_{n}(t) \in \Theta\left(S_{F, x_{n}}\right)$. Since $x_{n} \rightarrow x_{*}$, therefore, we have

$$
\begin{aligned}
\mathfrak{h}_{*}(t)= & -\int_{0}^{t} \frac{(t-s)^{q-1}}{\Gamma(q)} f_{*}(s) d s+\left(t-\frac{1}{2}+\frac{\xi-\eta}{\zeta}\right) \int_{0}^{1} \frac{(1-s)^{q-1}}{\Gamma(q)} f_{*}(s) d s \\
& +\int_{0}^{\frac{1}{2}} \frac{\left(\frac{1}{2}-s\right)^{q-1}}{\Gamma(q)} f_{*}(s) d s+\frac{\eta}{\zeta} \int_{0}^{1} \frac{(1-s)^{q-2}}{\Gamma(q-1)} f_{*}(s) d s
\end{aligned}
$$

for some $f_{*} \in S_{F, x_{*}}$.

In the last step, we discuss a priori bounds on solutions. Let $x$ be a solution of (1.1). Then there exists $f \in L^{1}([0,1], \mathbb{R})$ with $f \in S_{F, x}$ such that, for $t \in[0,1]$, we have

$$
\begin{aligned}
x(t)= & -\int_{0}^{t} \frac{(t-s)^{q-1}}{\Gamma(q)} f(s) d s+\left(t-\frac{1}{2}+\frac{\xi-\eta}{\zeta}\right) \int_{0}^{1} \frac{(1-s)^{q-1}}{\Gamma(q)} f(s) d s \\
& +\int_{0}^{\frac{1}{2}} \frac{\left(\frac{1}{2}-s\right)^{q-1}}{\Gamma(q)} f(s) d s+\frac{\eta}{\zeta} \int_{0}^{1} \frac{(1-s)^{q-2}}{\Gamma(q-1)} f(s) d s .
\end{aligned}
$$


In view of $\left(\mathrm{H}_{2}\right)$, for each $t \in[0,1]$, we obtain

$$
|x(t)| \leq\left[\frac{\left(2+\xi / \zeta+2^{-q}\right)}{\Gamma(q+1)}+\frac{\eta}{\zeta \Gamma(q)}\right]\|p\|_{\infty} \psi\left(\|x\|_{\infty}\right) .
$$

Consequently, it follows that

$$
\frac{M}{\psi(M)\|p\|_{\infty}}\left[\frac{\left(2+\xi / \zeta+2^{-q}\right)}{\Gamma(q+1)}+\frac{\eta}{\zeta \Gamma(q)}\right]^{-1} \leq 1 .
$$

By $\left(\mathrm{H}_{3}\right)$ there exists $M$ such that $\|x\|_{\infty} \neq M$. Let us set

$$
U=\left\{x \in C([0,1], \mathbb{R}):\|x\|_{\infty}<M\right\}
$$

Note that the operator $\Omega: \bar{U} \rightarrow \mathcal{P}(C([0,1], \mathbb{R}))$ is upper semicontinuous and completely continuous. From the choice of $U$, there is no $x \in \partial U$ such that $x \in \mu \Omega(x)$ for some $\mu \in$ $(0,1)$. Consequently, by Lemma 3.6 we deduce that $\Omega$ has a fixed point $x \in \bar{U}$, which is a solution of problem (3.1). This completes the proof.

\subsection{Nonconvex set-valued case}

In this subsection, we show the existence of solutions for the inclusion problem (3.1) with the right-hand side being nonconvex set-valued map by applying Wegrzyk's fixed point theorem. We first introduce the necessary background material.

Let $(\mathcal{X}, d)$ be a metric space, and $P_{b, c l}(\mathcal{X})=\{\mathcal{Y} \in \mathcal{P}(\mathcal{X}): \mathcal{Y}$ is bounded and closed .

Consider $H_{d}: \mathcal{P}(\mathcal{X}) \times \mathcal{P}(\mathcal{X}) \rightarrow \mathbb{R} \cup\{\infty\}$ given by

$$
H_{d}(A, B)=\max \left\{\sup _{a \in A} d(a, B), \sup _{b \in B} d(b, A)\right\}
$$

where $d(a, B)=\inf _{b \in B} d(a, b)$. The map $H_{d}$ is the (generalized) Pompeiu-Hausdorff functional. It is known that $\left(P_{b, c l}(\mathcal{X}), H_{d}\right)$ is a metric space and $\left(P_{c l}(\mathcal{X}), H_{d}\right)$ is a generalized metric space (see [33]).

Definition 3.10 A function $\delta: \mathbb{R}_{+} \rightarrow \mathbb{R}_{+}$is said to be a strict comparison function (see [29]) if it is continuous strictly increasing and $\sum_{n=1}^{\infty} \delta^{n}(t)<\infty$ for each $t>0$.

Definition 3.11 A multivalued operator $\mathcal{Q}: \mathcal{X} \rightarrow P_{c l}(\mathcal{X})$ is called

(a) $\varepsilon$-Lipschitz if and only if there exists $\varepsilon>0$ such that

$$
H_{d}(\mathcal{Q}(x), \mathcal{Q}(y)) \leq \varepsilon d(x, y) \quad \text { for each } x, y \in \mathcal{X}
$$

(b) a contraction if and only if it is $\varepsilon$-Lipschitz with $\varepsilon<1$;

(c) a generalized contraction if and only if there is a strict comparison function $\delta: \mathbb{R}_{+} \rightarrow \mathbb{R}_{+}$such that

$$
H_{d}(\mathcal{Q}(x), \mathcal{Q}(y)) \leq \delta(d(x, y)) \quad \text { for each } x, y \in \mathcal{X}
$$

Lemma 3.12 (Wegrzyk's fixed point theorem [34]) Let $(X, d)$ be a complete metric space. If $\mathcal{Q}: X \rightarrow P_{c l}(X)$ is a generalized contraction, then Fix $\mathcal{Q} \neq \emptyset$. 
Theorem 3.13 Assume that the following conditions hold:

$\left(\mathrm{H}_{6}\right) F:[0,1] \times \mathbb{R} \rightarrow P_{c p}(\mathbb{R})$ is such that $F(\cdot, x):[0,1] \rightarrow P_{c p}(\mathbb{R})$ is measurable for each $x \in \mathbb{R}$.

$\left(\mathrm{H}_{7}\right) H_{d}(F(t, x), F(t, \bar{x})) \leq \kappa(t) \delta(|x-\bar{x}|)$ for almost all $t \in[0,1]$ and $x, \bar{x} \in \mathbb{R}$ with a function $\kappa \in C\left([0,1], \mathbb{R}_{+}\right)$and $d(0, F(t, 0)) \leq \kappa(t)$ for almost all $t \in[0,1]$, where $\delta: \mathbb{R}_{+} \rightarrow \mathbb{R}_{+}$is strictly increasing.

Then problem (3.1) has at least one solution on $[0,1]$ if $\varepsilon \delta: \mathbb{R}_{+} \rightarrow \mathbb{R}_{+}$is a strict comparison function, where

$$
\varepsilon=\left(\frac{\left(2+\xi / \zeta+2^{-q}\right)}{\Gamma(q+1)}+\frac{\eta}{\zeta \Gamma(q)}\right)\|\kappa\|_{\infty}
$$

Proof Suppose that $\varepsilon \delta: \mathbb{R}_{+} \rightarrow \mathbb{R}_{+}$is a strict comparison function. It follows by assumptions $\left(\mathrm{H}_{6}\right)$ and $\left(\mathrm{H}_{7}\right)$ that $F(\cdot, x(\cdot))$ is measurable and has a measurable selection $v(\cdot)$ (see Theorem III.6 [35]). Also, $\kappa \in C([0,1], \mathbb{R})$ and

$$
\begin{aligned}
|v(t)| & \leq d(0, F(t, 0))+H_{d}(F(t, 0), F(t, x(t))) \\
& \leq \kappa(t)+\kappa(t) \delta(|x(t)|) \\
& \leq\left(1+\delta\left(\|x\|_{\infty}\right)\right) \kappa(t) .
\end{aligned}
$$

Thus, the set $S_{F, x}$ is nonempty for each $x \in C([0,1], \mathbb{R})$. Now we show that the operator $\Omega$ defined by (3.2) satisfies the assumptions of Lemma 3.12. To show that $\Omega(x) \in$ $P_{c l}((C[0,1], \mathbb{R}))$ for each $x \in C([0,1], \mathbb{R})$, let $\left\{u_{n}\right\}_{n \geq 0} \in \Omega(x)$ be such that $u_{n} \rightarrow u(n \rightarrow \infty)$ in $C([0,1], \mathbb{R})$. Then $u \in C([0,1], \mathbb{R})$, and there exists $v_{n} \in S_{F, x}$ such that, for each $t \in[0,1]$,

$$
\begin{aligned}
u_{n}(t)= & -\int_{0}^{t} \frac{(t-s)^{q-1}}{\Gamma(q)} v_{n}(s) d s+\left(t-\frac{1}{2}+\frac{\xi-\eta}{\zeta}\right) \int_{0}^{1} \frac{(1-s)^{q-1}}{\Gamma(q)} v_{n}(s) d s \\
& +\int_{0}^{\frac{1}{2}} \frac{\left(\frac{1}{2}-s\right)^{q-1}}{\Gamma(q)} v_{n}(s) d s+\frac{\eta}{\zeta} \int_{0}^{1} \frac{(1-s)^{q-2}}{\Gamma(q-1)} v_{n}(s) d s .
\end{aligned}
$$

Since $F$ has compact values, we pass onto a subsequence to obtain that $v_{n}$ converges to $v$ in $L^{1}([0,1], \mathbb{R})$. Thus, $v \in S_{F, x}$, and for each $t \in[0,1]$,

$$
\begin{aligned}
u_{n}(t) \rightarrow u(t)= & -\int_{0}^{t} \frac{(t-s)^{q-1}}{\Gamma(q)} v(s) d s+\left(t-\frac{1}{2}+\frac{\xi-\eta}{\zeta}\right) \int_{0}^{1} \frac{(1-s)^{q-1}}{\Gamma(q)} v(s) d s \\
& +\int_{0}^{\frac{1}{2}} \frac{\left(\frac{1}{2}-s\right)^{q-1}}{\Gamma(q)} v(s) d s+\frac{\eta}{\zeta} \int_{0}^{1} \frac{(1-s)^{q-2}}{\Gamma(q-1)} v(s) d s .
\end{aligned}
$$

Hence, $u \in \Omega(x)$.

Next, we show that

$$
H_{d}(\Omega(x), \Omega(\bar{x})) \leq \varepsilon \delta\left(\|x-\bar{x}\|_{\infty}\right) \quad \text { for all } x, \bar{x} \in C([0,1], \mathbb{R})
$$


Let $x, \bar{x} \in C([0,1], \mathbb{R})$ and $\mathfrak{h}_{1} \in \Omega(x)$. Then there exists $v_{1}(t) \in S_{F, x}$ such that, for each $t \in$ $[0,1]$,

$$
\begin{aligned}
\mathfrak{h}_{1}(t)= & -\int_{0}^{t} \frac{(t-s)^{q-1}}{\Gamma(q)} v_{1}(s) d s+\left(t-\frac{1}{2}+\frac{\xi-\eta}{\zeta}\right) \int_{0}^{1} \frac{(1-s)^{q-1}}{\Gamma(q)} v_{1}(s) d s \\
& +\int_{0}^{\frac{1}{2}} \frac{\left(\frac{1}{2}-s\right)^{q-1}}{\Gamma(q)} v_{1}(s) d s+\frac{\eta}{\zeta} \int_{0}^{1} \frac{(1-s)^{q-2}}{\Gamma(q-1)} v_{1}(s) d s .
\end{aligned}
$$

By $\left(\mathrm{H}_{7}\right)$ we have

$$
H_{d}(F(t, x), F(t, \bar{x})) \leq \kappa(t) \delta(|x(t)-\bar{x}(t)|) .
$$

So, there exists $w \in F(t, \bar{x}(t))$ such that

$$
\left|v_{1}(t)-w\right| \leq \kappa(t) \delta(|x(t)-\bar{x}(t)|), \quad t \in[0,1] .
$$

Define $V:[0,1] \rightarrow \mathcal{P}(\mathbb{R})$ by

$$
V(t)=\left\{w \in \mathbb{R}:\left|v_{1}(t)-w\right| \leq \kappa(t) \delta(|x(t)-\bar{x}(t)|)\right\} .
$$

Since the nonempty closed set-valued operator $V(t) \cap F(t, \bar{x}(t))$ is measurable (Proposition III.4 [35]), there exists a function $v_{2}(t)$ that is a measurable selection for $V(t) \cap$ $F(t, \bar{x}(t))$. So $v_{2}(t) \in F(t, \bar{x}(t))$, and for each $t \in[0,1]$, we have $\left|v_{1}(t)-v_{2}(t)\right| \leq \kappa(t) \delta(\mid x(t)-$ $\bar{x}(t) \mid)$.

For each $t \in[0,1]$, let us define

$$
\begin{aligned}
\mathfrak{h}_{2}(t)= & -\int_{0}^{t} \frac{(t-s)^{q-1}}{\Gamma(q)} v_{2}(s) d s+\left(t-\frac{1}{2}+\frac{\xi-\eta}{\zeta}\right) \int_{0}^{1} \frac{(1-s)^{q-1}}{\Gamma(q)} v_{2}(s) d s \\
& +\int_{0}^{\frac{1}{2}} \frac{\left(\frac{1}{2}-s\right)^{q-1}}{\Gamma(q)} v_{2}(s) d s+\frac{\eta}{\zeta} \int_{0}^{1} \frac{(1-s)^{q-2}}{\Gamma(q-1)} v_{2}(s) d s .
\end{aligned}
$$

Thus,

$$
\begin{aligned}
\left|\mathfrak{h}_{1}(t)-\mathfrak{h}_{2}(t)\right| \leq & \int_{0}^{t} \frac{(t-s)^{q-1}}{\Gamma(q)}\left|v_{1}(s)-v_{2}(s)\right| d s \\
& +\left|t-\frac{1}{2}+\frac{\xi-\eta}{\zeta}\right| \int_{0}^{1} \frac{(1-s)^{q-1}}{\Gamma(q)}\left|v_{1}(s)-v_{2}(s)\right| d s \\
& +\int_{0}^{\frac{1}{2}} \frac{\left(\frac{1}{2}-s\right)^{q-1}}{\Gamma(q)}\left|v_{1}(s)-v_{2}(s)\right| d s+\frac{\eta}{\zeta} \int_{0}^{1} \frac{(1-s)^{q-2}}{\Gamma(q-1)}\left|v_{1}(s)-v_{2}(s)\right| d s \\
\leq & \left(\frac{\left(2+\xi / \zeta+2^{-q}\right)}{\Gamma(q+1)}+\frac{\eta}{\zeta \Gamma(q)}\right)\|\kappa\|_{\infty} \delta\left(\|x-\bar{x}\|_{\infty}\right) .
\end{aligned}
$$

Hence,

$$
\left\|\mathfrak{h}_{1}-\mathfrak{h}_{2}\right\|_{\infty} \leq\left(\frac{\left(2+\xi / \zeta+2^{-q}\right)}{\Gamma(q+1)}+\frac{\eta}{\zeta \Gamma(q)}\right)\|\kappa\|_{\infty} \delta\left(\|x-\bar{x}\|_{\infty}\right) .
$$


Analogously, interchanging the roles of $x$ and $\bar{x}$, we obtain

$$
H_{d}(\Omega(x), \Omega(\bar{x})) \leq \varepsilon \delta\left(\|x-\bar{x}\|_{\infty}\right)=\left(\frac{\left(2+\xi / \zeta+2^{-q}\right)}{\Gamma(q+1)}+\frac{\eta}{\zeta \Gamma(q)}\right)\|\kappa\|_{\infty} \delta\left(\|x-\bar{x}\|_{\infty}\right)
$$

for all $x, \bar{x} \in C([0,1], \mathbb{R})$. Therefore, $\Omega$ is a generalized contraction. Thus, it follows by Lemma 3.12 that $\Omega$ has a fixed point $x$, which is a solution of (3.1). This completes the proof.

Remark 3.14 It is interesting to note that Theorem 3.13 holds for several values of the strictly increasing function $\delta: \mathbb{R}_{+} \rightarrow \mathbb{R}_{+}$. In particular, by choosing $\delta(y)=y$ in Theorem 3.13 we obtain its conventional Lipschitz form.

\subsection{Examples}

Consider the following multivalued boundary value problem

$$
\begin{aligned}
& { }^{c} D^{7 / 4} x(t) \in-F(t, x(t)), \quad t \in[0,1], \\
& x(0)=x(1), \quad \frac{3}{2} x^{\prime}(0)-\frac{1}{2} x^{\prime}(1)=\frac{1}{2} x(1 / 2),
\end{aligned}
$$

where $q=7 / 4, \xi=3 / 2, \eta=1 / 2, \zeta=1 / 2$. With the given data, we find that

$$
\frac{2+\xi / \zeta+2^{-q}}{\Gamma(q+1)}+\frac{\eta}{\zeta \Gamma(q)} \simeq 4.381671
$$

(a) Convex set-valued case. In this case, we choose $F(t, x)$ in (3.3) as

$$
F(t, x)=\left[\frac{|x|^{3}}{7\left(|x|^{3}+3\right)}+\frac{1}{12}\left[\sin ^{2}(t(1-t))+1\right], \frac{1}{3} e^{-t(1-t)} \cos x\right] .
$$

For $f \in F$, we have

$$
|f| \leq \max \left\{\frac{|x|^{3}}{7\left(|x|^{3}+3\right)}+\frac{1}{12}\left[\sin ^{2}(t(1-t))+1\right], \frac{1}{3} e^{-t(1-t)} \cos x\right\}, \quad x \in \mathbb{R}, t \in[0,1] .
$$

Thus,

$$
\|F(t, x)\|_{\mathcal{P}}:=\sup \{|y|: y \in F(t, x)\} \leq \frac{1}{3}, \quad x \in \mathbb{R},
$$

with $p(t)=1, \psi(\|x\|)=1 / 3$. Further, using condition $\left(\mathrm{H}_{3}\right)$, we find that $M>M_{1} \simeq 1.460557$. Clearly, all the conditions of Theorem 3.9 are satisfied. So there exists at least one solution of problem (3.3) with $F(t, x)$ given by $(3.5)$ on $[0,1]$.

(b) Nonconvex set-valued case. Let $F:[0,1] \times \mathbb{R} \rightarrow \mathcal{P}(\mathbb{R})$ in (3.3) be given by

$$
F(t, x)=\left[0, \frac{\tan ^{-1} x}{[3+t(1-t)]^{2}}+1 / 20\right]
$$

so that

$$
\sup \{|u|: u \in F(t, x)\} \leq 1 / 20+\frac{\pi}{2[3+t(1-t)]^{2}}
$$




$$
H_{d}(F(t, x), F(t, \bar{x})) \leq \frac{1}{[3+t(1-t)]^{2}}|x-\bar{x}|
$$

Fixing $\kappa(t)=\frac{1}{[3+t(1-t)]^{2}}$, we find that

$$
\varepsilon=\left(\frac{\left(2+\xi / \zeta+2^{-q}\right)}{\Gamma(q+1)}+\frac{\eta}{\zeta \Gamma(q)}\right)\|\kappa\|_{\infty} \simeq 0.486852
$$

and

$$
H_{d}(F(t, x), F(t, \bar{x})) \leq \kappa(t)|x-\bar{x}|
$$

where $\delta(y)=y$. Thus, all the conditions of Theorem 3.13 are satisfied, and hence its conclusion applies to problem (3.3) with $F(t, x)$ given by (3.6).

Remark 3.15 (An important consequence) In the limit $q \rightarrow 2^{-}$, our results correspond to the ones for a symmetric three-point second-order boundary value problem by taking the nonlinear functions $f(t, x)$ and $F(t, x)$ to be symmetric on $[0,1]$ and for all $x \in \mathbb{R}$. In [8], the authors discussed the symmetric positive solutions for a singular three-point boundary value problem. However, our (deduced) results in case of $q \rightarrow 2^{-}$are new.

Remark 3.16 Replacing the condition $\xi x^{\prime}(0)-\eta x^{\prime}(1)=\zeta x(1 / 2)$ with $\xi x^{\prime}(0)-\eta x^{\prime}(1)=\zeta x(\beta)$, $0<\beta<1$ in (1.1), we get a more flexible problem, which allows the unknown function $x$ to take its value at an arbitrary position $\beta \in(0,1)$. In this case, the integral solution of the resulting problem is

$$
\begin{aligned}
x(t)= & -\int_{0}^{t} \frac{(t-s)^{q-1}}{\Gamma(q)} f(s, x(s)) d s+\left(t-\beta+\frac{\xi-\eta}{\zeta}\right) \int_{0}^{1} \frac{(1-s)^{q-1}}{\Gamma(q)} f(s, x(s)) d s \\
& +\int_{0}^{\beta} \frac{(\beta-s)^{q-1}}{\Gamma(q)} f(s, x(s)) d s+\frac{\eta}{\zeta} \int_{0}^{1} \frac{(1-s)^{q-2}}{\Gamma(q-1)} f(s, x(s)) d s .
\end{aligned}
$$

Existence results for this new nonlocal problem can be obtained by following the procedure used in Sections 2 and 3.

\section{Competing interests}

The authors declare that they have no competing interests.

\section{Authors' contributions}

Each of the authors, BA, RPA, and AA contributed to each part of this work equally and read and approved the final version of the manuscript.

\section{Author details}

'Nonlinear Analysis and Applied Mathematics (NAAM) Research Group, Department of Mathematics, Faculty of Science, King Abdulaziz University, P.O. Box 80203, Jeddah, 21589, Saudi Arabia. ${ }^{2}$ Department of Mathematics, Texas A\&M University, Kingsville, TX 78363-8202, USA.

\section{Acknowledgements}

This article was funded by the Deanship of Scientific Research (DSR), King Abdulaziz University. The authors, therefore, acknowledge with thanks DSR technical and financial support. 


\section{References}

1. Webb, JRL, Lan, KQ: Eigenvalue criteria for existence of multiple positive solutions of nonlinear boundary value problems of local and nonlocal type. Topol. Methods Nonlinear Anal. 27, 91-116 (2006)

2. Clark, S, Henderson, J: Uniqueness implies existence and uniqueness criterion for non local boundary value problems for third-order differential equations. Proc. Am. Math. Soc. 134, 3363-3372 (2006)

3. Wang, SJ, Sun, HR: Optimal existence criteria for symmetric positive solutions to a singular three-point boundary value problem. Nonlinear Anal. 69, 4266-4276 (2008)

4. Webb, JRL, Infante, G, Franco, D: Positive solutions of nonlinear fourth order boundary value problems with local and nonlocal boundary conditions. Proc. R. Soc. Edinb., Sect. A 138, 427-446 (2008)

5. Graef, JR, Henderson, J, Ouahab, A: Differential inclusions with nonlocal conditions: existence results and topological properties of solution sets. Topol. Methods Nonlinear Anal. 37, 117-145 (2011)

6. Akyildiz, FT, Bellout, H, Vajravelu, K, Van Gorder, RA: Existence results for third order nonlinear boundary value problems arising in nano boundary layer fluid flows over stretching surfaces. Nonlinear Anal., Real World Appl. 12, 2919-2930 (2011)

7. Horodetskyi, WV, Martynyuk, OV, Petryshyn, Rl: Correct solvability of a nonlocal multipoint (in time) problem for one class of evolutionary equations. Ukr. Math. J. 65, 377-392 (2013)

8. Zhou, J: Global existence and blowup for a degenerate and singular parabolic system with nonlocal source and absorptions. Z. Angew. Math. Phys. 65, 449-469 (2014)

9. Infante, G, Pietramala, P, Tenuta, M: Existence and localization of positive solutions for a nonlocal BVP arising in chemical reactor theory. Commun. Nonlinear Sci. Numer. Simul. 19, 2245-2251 (2014)

10. Benchohra, M, Hamani, S, Ntouyas, SK: Boundary value problems for differential equations with fractional order and nonlocal conditions. Nonlinear Anal. 71, 2391-2396 (2009)

11. Zhong, W, Lin, W: Nonlocal and multiple-point boundary value problem for fractional differential equations. Comput. Math. Appl. 39, 1345-1351 (2010)

12. Webb, JRL, Infante, G: Semi-positone nonlocal boundary value problems of arbitrary order. Commun. Pure Appl. Anal. 9, 563-581 (2010)

13. Ahmad, B, Ntouyas, SK, Alsaedi, A: New existence results for nonlinear fractional differential equations with three-point integral boundary conditions. Adv. Differ. Equ. 2011, Article ID 107384 (2011)

14. Ahmad, B, Agarwal, RP: On nonlocal fractional boundary value problems. Dyn. Contin. Discrete Impuls. Syst., Ser. A Math. Anal. 18, 535-544 (2011)

15. Ahmad, B, Sivasundaram, S: On four-point nonlocal boundary value problems of nonlinear integro-differential equations of fractional order. Appl. Math. Comput. 217, 480-487 (2010)

16. Henderson, J, Luca, R: Positive solutions for a system of nonlocal fractional boundary value problems. Fract. Calc. Appl. Anal. 16, 985-1008 (2013)

17. Ahmad, B, Nieto, JJ, Alsaedi, A, Al-Hutami, H: Existence of solutions for nonlinear fractional q-difference integral equations with two fractional orders and nonlocal four-point boundary conditions. J. Franklin Inst. 351, $2890-2909$ (2014)

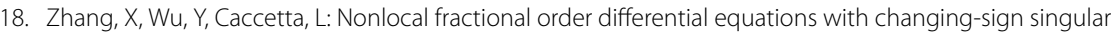
perturbation. Appl. Math. Model. 39, 6543-6552 (2015)

19. Binlin, Z, Bisci, GM, Servadei, R: Superlinear nonlocal fractional problems with infinitely many solutions. Nonlinearity 28, 2247-2264 (2015)

20. Ahmad, B, Ntouyas, SK: Nonlocal fractional boundary value problems with slit-strips boundary conditions. Fract. Calc. Appl. Anal. 18, 261-280 (2015)

21. Samko, SG, Kilbas, AA, Marichev, Ol: Fractional Integrals and Derivatives. Theory and Applications. Gordon \& Breach, Yverdon (1993)

22. Kilbas, AA, Srivastava, HM, Trujillo, JJ: Theory and Applications of Fractional Differential Equations. North-Holland Mathematics Studies, vol. 204. Elsevier, Amsterdam (2006)

23. Mainardi, F: Fractional Calculus and Waves in Linear Viscoelasticity: An Introduction to Mathematical Models. Imperial College Press, London (2010)

24. Klafter, J, Lim, SC, Metzler, R (eds.): Fractional Dynamics in Physics. World Scientific, Singapore (2011)

25. Baleanu, D, Diethelm, K, Scalas, E, Trujillo, JJ: Fractional Calculus Models and Numerical Methods. Series on Complexity, Nonlinearity and Chaos. World Scientific, Boston (2012)

26. Covitz, H, Nadler, S: Multivalued contraction mappings in generalized metric spaces. Isr. J. Math. 8, 5-11 (1970)

27. Lan, KQ, Lin, W: Positive solutions of systems of Caputo fractional differential equations. Commun. Appl. Anal. 17, 61-85 (2013)

28. Smart, DR: Fixed Point Theorems. Cambridge University Press, Cambridge (1980)

29. Granas, A, Dugundji, J: Fixed Point Theory. Springer, New York (2003)

30. Deimling, K: Multivalued Differential Equations. de Gruyter, Berlin (1992

31. Hu, S, Papageorgiou, N: Handbook of Multivalued Analysis. Vol. I. Theory. Kluwer Academic, Dordrecht (1997)

32. Lasota, A, Opial, Z: An application of the Kakutani-Ky Fan theorem in the theory of ordinary differential equations. Bull. Acad. Pol. Sci., Sér. Sci. Math. Astron. Phys. 13, 781-786 (1965)

33. Kisielewicz, M: Differential Inclusions and Optimal Control. Kluwer Academic, Dordrecht (1991)

34. Wegrzyk, R: Fixed point theorems for multifunctions and their applications to functional equations. Diss. Math. 201, $28(1982)$

35. Castaing, C, Valadier, M: Convex Analysis and Measurable Multifunctions. Lecture Notes in Mathematics, vol. 580. Springer, Berlin (1977) 